\title{
The impact of Lung-Strengthening Qigong on wellbeing: a case study
}

\author{
Zeyneb Kurt ${ }^{1 *}$, Petia Sice ${ }^{1}$, Krystyna Krajewska ${ }^{2}$, Garry Elvin ${ }^{1}$, Hailun Xie ${ }^{1,3}$, Suzannah Ogwu ${ }^{1,4}$, \\ Pingfan Wang ${ }^{1}$, Sultan Sevgi Turgut ${ }^{5}$ \\ ${ }^{1}$ Department of Computer and Information Sciences, University of Northumbria, Newcastle \\ Upon Tyne, UK \\ ${ }^{2}$ Confucius Institute, University of Wales Trinity Saint David, Lampeter, Ceredigion, UK \\ ${ }^{3}$ Renewable Energy Group at the University of Exeter, Penryn Campus, Penryn, Cornwall, UK \\ ${ }^{4}$ University of Wales Trinity Saint David, Birmingham, Sparkhill Centre, UK \\ ${ }^{5}$ Department of Computer Engineering, Yildiz Technical University, Istanbul, Turkey \\ ${ }^{*}$ Corresponding author: zeyneb.kurt@northumbria.ac.uk
}

\begin{abstract}
Qigong is an umbrella term for a group of traditional exercises originated from China. LungStrengthening Qigong (LSQ) is one of these techniques enabling practitioners to maintain and improve their physical and mental wellbeing. We recruited 170 practitioners and 42 nonpractitioner/control samples to investigate the impacts of LSQ practice on body, mind, thoughts, and feelings. We requested completion of a questionnaire regularly from both of the practitioner and control group, fill in an online diary and end of study survey (EOS) only from the practitioners. Statistical analysis was conducted on the questionnaires, whereas qualitative thematic- and quantitative machine learning-based analyses were applied to the free-text diary entries. We evaluated all different data resources together and observed that (a) there was a significant improvement in physical and mental wellbeing (increase in sleep quality, feeling life, coping with life, feeling life energy and a decline in stress amount) of the practitioners, which were not observed in the control group, (b) four different groups (non, low, moderate, high-level) of benefits were emanated among the practitioners, (c) numerical evaluation of questionnaires and EOS, as well as the qualitative and quantitative analyses of the diary entries were all found to be consistent, and (d) majority of the participants (84\%) reported a striking improvement in their well-being, (e) majority of the positively impacted practitioners had no or some little prior experience with LSQ. This study is novel in various aspects including (i) increasing the sample size radically compared to other conventional studies as well as considering a control group for comparisons, (ii) providing regular live LSQ sessions to the practitioners, (iii) incorporating both qualitative and quantitative type of analyses to understand the impacts of Qigong.
\end{abstract}




\section{Introduction}

Qigong embraces a family of self-care exercises originating in China several thousand years ago and are considered in much of Asian culture as a normal aspect of personal health maintenance. The term was coined in China in the mid-20th Century and consists of qi (气), frequently translated as 'energy' (Kohn, 1993) and gong (功) work. Qigong literally means, exercise for working with qi energy.

Existing studies have shown that Qigong practice offers a wide range of benefits, including alleviating various health conditions, such as asthma, hypertension, and diabetes, preventing psychosomatic and respiratory infectious diseases [1], [2], providing significant therapeutic benefits for cancer patients [3]-[8], and strengthening immune system. Qigong practitioners experience a significant increase in the number of white blood cells and lymphocytes [9], [10] and an evident decrease in total cholesterol, blood pressure [11], [12], as well as improvements in depression scores [13]-[15]. Qigong can also complement the treatment of cognitive impairments, mood and mental health conditions [13], [16]. However, existing studies suffer from a number of limitations, such as small sample sizes (less than 30) [9], [10], [12], substantial reliance on quantitative analysis (e.g. measuring bodily functions and symptoms, immune cell count, blood pressure, cardiac function) [10], [11], [17]-[21], as well as lack of control samples for comparison [22]-[24].

In recent decades a gradual move in China to incorporate Traditional Chinese Medicine (TCM) within a scientific framework has resulted in the development of Medical Qigong that focuses more closely on physiology and on prescribing a particular practice for specific health conditions. Lung-Strengthening Qigong (LSQ), which is the focus of our study, is one typical example.

The LSQ exercise employed in this study was designed by Jiangxi University of Chinese Medicine during the early months of the Covid-19 pandemic in 2020, and was prescribed for supporting lung resilience, constitutional robustness, and general wellbeing. The underlying principle of the exercise is to manipulate Qi energy and direct it to specific areas of the body using mostly slow arm movements. It is carried out in a standing position while maintaining sustained focus on the physical movements.

Despite extensive studies conducted to explore various benefits of common Qigong practice, a literature review indicates no prior investigations into LSQ. Therefore, in this study we investigated the impact of LSQ practice on (i) physical symptoms; (ii) mental wellbeing; (iii) connection to nature and others, and (iv) relational and life-energy awareness. Quantitative and qualitative analyses were conducted on the data collected from 170 participants by survey, questionnaire, and diary entries. We also recruited 42 people as a control group for comparison. Our study fills the gaps in the literature by: (i) encompassing both quantitative analysis (based on a statistical and machine learning-based semantic analysis of surveys and diary entries) and qualitative analysis (based on thematic analyses completed in NVIVO software and manual classification of diary entries); (ii) recruiting a larger number of participants (170 practitioners) in contrast with the small sample size (typically around 30) commonly employed in existing studies; (iii) incorporating 42 control users; (iv) grounding our perspective in systems theory. 


\section{An Integrative Model of Wellbeing}

An integrative model of wellbeing was used in this study (as shown in Figure 1). The model was previously used to evaluate wellbeing among staff and patients in public health services [25], [26]. An integrated view of the processes in a human living system consists of the following components:

- Body - physiological processes and systems working together, e.g., immune system, nervous system, and endocrine system.

- Mind - mental activities and processes, e.g., thoughts, images, feelings, concepts, and narratives.

- Relational processes - processes of mutual coupling with the environment, e.g., interaction with other living beings, nature, artefacts.

- Awareness - processes of becoming aware of sensory, somatic, mental and relational experiences.

The human system acts and evolves with its environment [27]-[30]. Porges suggests that humans first orient themselves and then act [31]. This is interpreted in the model (Figure 1) as valence, that is, a positive or negative disposition that arises from the complex interrelated processes of body, mind, and awareness within the environment of the individual. Thus, wellbeing may be defined as a state of body, mind, relational and awareness coherence [26], [32]. This definition is consistent with the biological systems perspective of wellbeing as a coherent and energised state of functioning [33]. A coherent state is one in which each component of the system is differentiated and at the same time integrated in the whole [33], [34]. An energised state is characterised by the capacity of the system to adapt, respond and evolve [33]-[35]. Multiple research studies provide evidence that the physical, mental, relational, and awareness processes are in reciprocal dynamic relationship, and impact wellbeing (Suppl Table S1). Therefore, in this study we measured the four pillars of the integrative model of wellbeing, i.e., body, mind, relational processes, and awareness, for the LSQ participants.

\section{Background and design of data collection}

Data collected from first-person accounts is essential for understanding the characteristics of experience [36]-[38]. Thus, in our study an online diary was developed to capture participants' immediate experience after each practice, structured by prompts mirroring features of the LSQ exercise (Suppl Figure S1). At the end of the study (EOS), participants were asked to record their experiences and indicate perceived changes in physical, mental and relational wellbeing (Suppl Figure S2). In addition to the diary and the EOS survey, a Baseline Self-assessment Questionnaire was designed to capture behavioral aspects of change in participants' well being (Suppl Figure S3). The questionnaire was completed at the beginning, mid and endpoint of the study. The design of all data collection tools was informed by the integrative model of wellbeing. Thus, the Diary Entries (DE), the Baseline Questionnaire (BQ) and the End of Study Survey (EOS) were designed to capture changes relating to the physical, mental, awareness, and relational domains with reference to the research literature (Suppl Table S1). 
The Baseline Questionnaire included questions relating to the frequency with which participants experienced different states that undermined or supported their wellbeing (Suppl Figure S3). Numerical scales [39] were used for all questions to capture the responses, excluding question BQ4, where radio buttons were used for specific symptoms (Suppl Table S1). A scale of 1-100 (implemented in a slider) was used to capture a high granularity of detail in the responses (as suggested by [39], [40]) for a group of questions (Suppl Table S1). To ensure that the participants engage mindfully and reflect on their response, a counterintuitive reversal of the positive scale questions (so that a lower score shows improvement) was deployed [41]. It is important to emphasise that the quantitative data was triangulated with the diary and the EOS text data to cross-reference the meaning participants attributed to the sliders [39].

The research questions explored in this study are: (i) What is the impact of LSQ on physical, mental and relational wellbeing? (ii) What are the characteristics of the participants' experiences of the LSQ practice? (iii) How does the LSQ practice impact the participants' awareness of their mental, physical states, and relational states? Our goal was to test particular hypotheses to answer these questions by performing quantitative and qualitative analyses using the data collected:

Hypothesis-1: The LSQ practice enhances practitioners' physical, mental, and relational wellbeing compared to the control group (null hypothesis states that there is no difference between the practicing and control groups).

Hypothesis-2: LSQ enhances the participants' awareness of their environment, body, mental activity, and perception of Qi compared to the control (null hypothesis states that there is no difference between the two groups).

Hypothesis-3: All participants perceive the same level of effectiveness from LSQ, regardless of variations in prior experiences (e.g. non-experienced, beginner, advanced-level) (null hypothesis).

\section{Results}

In total, 170 Qigong practice volunteers and 42 control volunteers were recruited. In the practice group, 72 participants regularly logged entries in the online diary and completed the baseline and follow-up questionnaires as required by the study. Among 42 control samples 13 users completed the questionnaire (the number of the questionnaire entries and participants who filled the Baseline Questionnaire (BQ) is detailed in Suppl Table S2). As the control group did not participate in the LSQ exercise they did not have access to the online diary. Three types of analysis (quantitative, semantic, and qualitative), were conducted on the collected diary and questionnaire data to evaluate the dynamic responses of participants throughout their LSQ practice journey. The results indicate that the practice has a statistically significant positive impact on the wellbeing of participants. Using Machine Learning (ML) algorithms the efficacy of LSQ practice was automatically categorised into four clusters: 1) no change, 2) low change, 3) moderate change, 4) high level of change, thus accounting for individual differences in receptiveness to the practice. 
Among the 72 participants in the practice cohort, those aged 65-74 completed the baseline questionnaire more often than participants in the other age groups over the whole 8-week period (Figure 2A). Completion by the age groups 35-44, 45-54, and 55-64 was almost the same. Three-quarters of the participants were women (Figure 2B). More than one-third of participants declared they had some prior experience of Qigong, and more than one-quarter had no prior experience (Figure 2C). In the control group, the age group 35-44 was found to have largest sample size among the participants (more than one-third, Figure 2A). The majority of the participants were women (more than one-third, Figure 2B) and had no prior experience of Qigong practice (more than one-third, Figure 2C). Other demographic details, including work status and ethnic heritage of the participants are provided in Figure 2D and E, respectively. The majority of the practitioners were either retired (38\%) or had another occupation $(30 \%)$, whereas in the control group occupations were almost uniformly distributed. Lastly, the majority of the participants were from a white ethnic background (85\% and $100 \%$ for the practitioner and control groups respectively).

\section{Comparison of participant and control group baseline and follow-up questionnaires highlights benefits of Qigong practice}

Both the practitioner and control groups were asked to complete the baseline questionnaire at the beginning, midpoint, and end of the study. The questions and associated scoring schemes are summarised in Suppl Table S2. The scores for questions, scaled between 1-100, were analysed both individually and pooled together across three timepoints. Similarly, score changes in items scaled between 1-4 were tracked together as well as individually.

We observed a statistically significant improvement over time in the scores of the LSQ practitioners for questionnaire items scaled 1-100 (from initial point to the midpoint [l2M] of the study $P=0.0016$, from midpoint to endpoint [M2E] $P=6.6 \mathrm{E}-5$, and from initial to endpoint [I2E] $P=3 \mathrm{E}-12$, Figure $3 \mathrm{~A})$. Whereas in the control group, with no LSQ intervention, there is no statistically significant change in the scores across the three timepoints of our study (from I2M $P=0.18$, from M2E $P=0.61$, from I2E $P=0.29$, Figure 3B). As detailed in Methods, a decrease in the questionnaire scores reflects an improvement. For the items scaled 1-4, there is no significant change in scores over time in either the practice or control groups, except for a significant improvement in the practitioner group from the I2E of the study $(P=0.015$, Figure $3 \mathrm{C}$, 3D). These items are related to health conditions such as muscle pain, headache, and coughing (Suppl Table S2). An increase in scores means a less severe health condition.

In individual analyses of the questionnaire items, scaled 1-100, we observed a significant improvement over time in the practice group for most of the items (Figure 4), including sleep (from I2E $P=0.019$ ), waking up calmly (I2E $P=0.0081$ ), emotional life (I2E $P=0.0096$ ), coping with life (I2E $P=0.039$ ), positive feeling towards life (I2E $P=0.0022$ ), connection to others (I2E $P=0.013$ ), life energy (I2E $P=0.011$ ), and levels of stress (I2E $P=0.019$ ). Despite the lack of a statistically significant outcome $(P>0.05)$ for the remaining items, there is a clear trend of improvement in the scores (especially from I2E) as highlighted in Figure 4A-P. In the individual analysis of the questions scaled 1-4, there is no statistically significant change in the practice 
group, yet there is an obvious trend of improvement observed in almost all items, especially in muscle and joint pain, digestive problems, headaches, and fatigue (Figure 4Q-W).

The same analysis was performed for the control group. We observed no significant improvement or any particular trend for either the questionnaire items scaled 1-100 or those scored 1-4 (Suppl Figure S4A-W). Hence, a significant improvement was observed in multiple aspects for the practitioner group, whereas no significant improvement (neither trends nor indicators) in the scores for the control group was found. Mean score changes in questionnaire items according to the three timepoints for both practitioner and control groups are also demonstrated as radar graphs in Suppl Figure S5A-D. Radar graphs representing mean score changes for both the practitioner and control groups can be found in Suppl Figure S5A-D.

\section{Questionnaires reveal that specific demographic groups derive greater benefit from Qigong practice}

We analysed the questionnaire score changes of the practice group in detail, based on the different demographic subgroups such as age, previous experience of Qigong, and gender. The majority of the age groups (35-44, 45-54, 55-64, and 65-74) in the practice cohort demonstrated a notable trend towards improvement in their scores over time (Suppl Figure S6A-B), whereas the control group did not show any such improvement in any age group (Suppl Figure S6C-D). Score improvement was found in both male and (especially) female practice volunteers whereas no significant changes or trends were observed for the control group (Suppl Figure S6E-H).

Subgroups with no prior experience of Qigong, some prior experience, and prior experience of Tai Chi among the practice group demonstrated the most significant and distinct improvement, whereas no significant change or trends were observed in the control group for any experience level (Suppl Figure S6I-L). The improvements observed in the practice group as a whole (see Figure 4) were consistent in the majority of the subcategories in age groups, in both genders, and in three of the prior experience levels (no experience, some prior Qigong, and Tai Chi experience). In contrast, control samples (both as a whole, and at the sub-category level), did not demonstrate any significant changes in their scores.

\section{Significant concordance exists between the questionnaire items}

We analysed the correlation between the questionnaire items in a pairwise manner to understand which items show similar change over time when paired, and to assess the impact of LSQ from multiple dimensions at a single glance. We observed that five items, coping with life, emotional life, stress, positive feeling towards life, and positive feelings towards self, demonstrated strong positive correlations with one another (Spearman correlation scores between 0.60 and 0.72 , Suppl Figure S7). Since the questionnaire scores for the majority of these items significantly improved and changed jointly in the same (positive or negative) direction throughout the journey of the LSQ study, we can infer that they are highly associated and improve jointly over time for the practice group. Kindness, awareness of thoughts-emotions, judgement, sensory-somatic awareness, life energy, and connection to nature, are also positively correlated (Spearman coefficients between 0.40-0.64). The first set of items that were observed as changing together relate to self-awareness and emotions concerning coping with 
life, whereas the second cluster of items concerns feelings towards others or to the environment (such as nature). Lastly, we observed a moderate to high correlation (scores within [0.25-0.50]) between the items: headaches, digestive problems, joint pain, muscle pain, and fatigue (scores within [0.4-0.5]). This indicates that particular health conditions of the practitioners (such as headaches, joint or muscle pain, and fatigue) are highly associated. Fatigue was also found to be highly correlated with the items cough and shortness in breath (correlation scores 0.41 and 0.47 respectively, Suppl Figure S7).

\section{Analysis of diary entries reveals four different clusters of Qigong experience}

Participants were asked to complete regular diary entries. Diary fields for each dimension (attribute) of the Integrative Wellbeing model were provided (see Introduction): (a) body awareness, (b) focused attention, (c) Qi energy awareness, (d) relational awareness, (e) satisfaction/meaning. On average, each participant completed the diary twice a week and 15.24 times across the 8-week period of the practice. We checked whether particular patterns or levels of benefit from LSQ experience occurred among participants based on their selfreporting. Text data derived from the diary entries was converted into Term Frequency-Inverse Document Frequency (TF-IDF) statistics (see Methods). Then, $k$-means clustering (see Methods) was employed to uncover the underlying patterns embedded in the TF-IDF scores. The elbow method was applied to identify the optimal number of clusters, which was found to be 4 (see Methods, Suppl Figure S8).

Hence, four different types of patterns are found in the diary entries, representing four distinct levels of benefit from LSQ practice, i.e., 1) no change, 2) low change, 3) moderate change, 4) high level of change. Each cluster is driven by a particular set of sentiments and reflects a unique type of LSQ journey. Word clouds for clusters 1,2,3,4 can be found in Figure 5A-D respectively. Additionally, the top 100 words by frequency of occurrence in each cluster are listed in Suppl Table S3. Values of the central points of each cluster across the five diary dimensions, as well as the number of the entries in each cluster are summarized in Table 1. Centroid of cluster $1=\{0.167,0.175,0.0338,0.185,0.446\}$, cluster $2=\{0.172,0.207,0.448,0.250$, $0.263\}$, cluster $3=\{0.205,0.296,0.0455,0.101,0.00\}$, and cluster $4=\{0.037,-0.031,0.030,0.039$, $0.017\}$ for the attributes (a)-(e) listed above, respectively; and there were 206, 87, 773, 199 entries that were assigned to clusters $1,2,3$, and 4 respectively. Weekly changes to the number of samples that are assigned to each cluster are given in Suppl Table S4. The top 100 most frequent words of each cluster were used to run a sentiment analysis to identify the positivity of the clusters (see Methods). In cluster 1, terms such as tension, tiredness, no change, and difficulty, occurred frequently and, on the basis of sentiment analysis were predicted as relating to a pattern of no change. In cluster 2, words such as calm, improvement and concentration were highly represented, whereas cluster 3 included the terms satisfaction, energy, focused, and feel, and cluster 4 extensively contained words such as connected, warm, and better. Clusters 1, 2, 4, and 4 were predicted as corresponding to (i) no, (ii) low, (iii) moderate, and (iv) high-level of perceived change (and benefit gained) respectively.

In addition to the natural language processing (NLP)-based analysis of the topmost frequently utilized words, a manual examination of the entries also confirmed that these four clusters exist and range from none to a high level of perceived change. Examples of the diary entries can be found in Suppl Table S5. Based on narratives and frequently used vocabulary, users from the low-level change group (cluster-2) improved only in physical aspects; whereas the cluster for 
moderate-level (cluster-3) change reflects improvement in both physical and mental spheres. In contrast, the high-level perceived change group(cluster-4) demonstrates more intensely experienced benefits resulting from the exercise.

Lastly, among the 83 participants who regularly completed the diary, the entries of 70 practitioners were predicted as occurring in the moderate or high-level of change clusters. Hence, the majority of the participants $(70 / 83 \sim 84 \%$ ) benefitted highly from the study (Suppl Table S6).

\section{Qualitative data analysis of diary entries is consistent with the findings from NLP analysis}

The participants' entries were read and coded into subthemes. The subthemes identified specific aspects of individual experience (Suppl Figure S9A-F). 933 of the diary entries data were coded, according to specific types of experience within the five themes (Suppl Figure S9A):

- Body awareness - experiences were related to breathing, relaxation, discomfort, specific body sensations, emotions, and balance (Suppl Figure S9B). Reported qualities of breathing include the words: deep, slow, calm, easier breathing (Suppl Figure S9C). Most specific body sensations were described as feeling warmth, feeling grounded and sensations of 'pins and needles' (Suppl Figure S9D). Participants from clusters 3 and 4 consistently reported feeling relaxed (16.69\% coverage of bodily awareness - there were 116 entries, Suppl Figure S9B). Predominant emotions in clusters 3 and 4 were peace, love, and joy. Some participants reported an improved balance (1.7\% coverage of bodily awareness - 12 entries, Suppl Figure S9B). A small number of entries (81 entries, $11.65 \%$ coverage of bodily awareness) described sensations of discomfort related mainly to pain and tiredness (Suppl Figure S9E).

- Focused attention - the predominant reported experiences were steady focus, feeling calm, clarity and ability to visualise. A smaller number of entries described difficulty focusing (74 references, $14.34 \%$ coverage of focus awareness) and a fluctuating focus (38 references, 7.36\% coverage of focused awareness) (Suppl Figure S9F).

- Qi awareness - most participants (89 references, 22\% coverage of Qi awareness) perceived Qi as energy (Suppl Figure S9H). The feeling of Qi was described as the sensation of tingling and pulsing, feeling, or touching energy, and effortless action (Suppl Figure $\mathrm{S9H}$ ).

- Relational awareness - the majority of entries (281 entries, $63.13 \%$ coverage of the satisfaction and meaning awareness) reported feelings of connection to body, self, nature while some entries reported connection to others, with a small number of entries reporting no connection at all (89 entries, $23.6 \%$ coverage of the relational awareness (Suppl Figure S9I). The experience of connection was described as connection to self, nature, and others, with a number of entries (55 entries, $12.35 \%$ of relational awareness) reporting a sense of unity, being larger than the self, unity of self and universe (Suppl Figure S9I)

- Satisfaction and meaning - The theme of satisfaction and meaning was articulated as an increased sense of satisfaction and wellbeing, self-care, self-empowerment, sense of meaning, gratitude and compassion, improved coping with life. A large number of entries (115 entries and $39.65 \%$ coverage of satisfaction and meaning) reported learning, new 
understanding and increased curiosity (Suppl Figure S9J). A small number of entries (23 entries and $7.93 \%$ coverage) reported no change (Suppl Figure S9J).

\section{Qualitative data analysis of End of study (EOS) survey text entries}

The End of Study questionnaire included the opportunity for participants to list benefits they experienced linked to their practice of LSQ. These include better physical wellbeing: improved breathing, less tension, relaxed body, stronger and a more energised core, better balance; improved mental wellbeing: feeling calmer, more positive, feeling grounded, experience of peace; more focus; better relational wellbeing: better body awareness and connection to self; connection to nature, feeling of being part of a whole bigger than self; sense of satisfaction and meaning: sense of achievement, new knowledge, sense of gratitude, improved understanding, and awareness of qi; better overall wellbeing: improved quality of life, self-care and health awareness (Suppl Figure S10, "List of benefits generated by Nvivo"). Participants were generally surprised with the benefits they experienced as their prior expectations were mainly restricted to developing new knowledge, having a pleasant experience and willingness to help (Suppl Figure S10).

\section{End of study (EOS) survey supports the findings from the baseline and follow-up questionnaires as well as the diary entries}

We compared our findings from three different self-evaluation sources consisting of (i) baseline questionnaires from all three time points, (ii) text data from the diary, and the (iii) end of study (EOS) survey. The EOS survey was completed by 63 practice group participants. Of these, 59 had sufficient data from all sources: (i) completion of the questionnaires at all three time points, (ii) at least three diary entries, (iii) EOS survey. Some of these participants (15 among 59) did not fall into any of the categories of Pseudo True Positive (PTP), Pseudo True Negative (PTM), Pseudo False Positive (PFP), and Pseudo False Negative (PFN) as explained in the Methods, but most of them (44 among 59, i.e., 75\%) fitted these categories and were classified according to the $\{P T P, P T N, P F P, P F N\}$ labels (Table 2 ). The majority (28 among 44, Table 2 ) were found to reflect a highly positive experience according to their diary entries (entries were in clusters $\{3,4\}$ ) and EOS survey results (a score $>400$ ). Additionally, a significant improvement across time was observed for the majority of the items queried in the baseline questionnaire. Hence, we observed consistent improvements and positive feedback from $64 \%$ (28/44) of the practitioners. For example, changes of the baseline questionnaire scores of four randomly selected participants among the PTP samples (see Methods, Table 2) are demonstrated in Figure $6 A-D$, for users with the IDs $56,100,102,157$, respectively. Only five practitioners among the 28 highly satisfied participants had previously practiced Qigong to a professional level; the remainder either had no experience or some prior experience. Only five participants among $44(11 \%)$ declared low EOS survey scores $(<200)$ and their entries were consistently clustered in clusters 1 and 2 (no change or low-level change). Furthermore, their baseline questionnaire scores were not found to have changed or improved over time. As an example, changes of the baseline questionnaire scores of four of the PTN samples are demonstrated in Figure 6E-H, for user IDs 45, 73, 79,112, respectively. Inconsistency between the diary, EOS survey, and questionnaires was observed for a small set of samples. Among 44, only five practitioners (11\%) gave a high EOS survey score while their diary entries reflected no (or lowlevel) change, which were labeled as PFN; whereas six samples (14\%) gave a low EOS survey score while their entries were clustered as reflecting a high level of benefit/changes (PFPs). As a result, the majority of the 44 practitioners that could be labeled as one of the classes $\{P T P$, 
PTN, PFP, PFN\}, were found to be in the PTP class (28/44=64\%), whose diary entries, baseline questionnaires, and EOS survey highlight a consistent and positive improvement and awareness altogether.

\section{Discussion}

The study rejected all of the null hypotheses stated in $\mathrm{H} 1$ (hence, there is strong evidence for improvement in physical, mental, and relational wellbeing of practitioners compared to the control); $\mathrm{H} 2$ (thus there is evidence the LSQ program enhances the practitioners' awareness of their environment, body and mental state, and Qi compared to the control), and H3 (hence, there is a difference between the non-experienced/beginner and advanced-level practitioners in terms of the benefits highlighted in $\mathrm{H} 1$ and $\mathrm{H} 2$ ). We were expecting the rejection of the null hypotheses in $\mathrm{H} 1$ and $\mathrm{H} 2$ (this was confirmed by the results), meanwhile anticipating a failure of rejection of the null hypothesis in $\mathrm{H} 3$. Overall, the rejection of all three null hypothesis indicates that LSQ is evidently capable of elevating people's wellbeing and their awareness of it. Moreover, the less prior experience with Qigong, the greater the benefits that people are likely to obtain from LSQ. All three sets of data (BQ, DE, EOS survey), were triangulated to explore these hypotheses.

The BQ data analysis showed enhanced physical wellbeing, i.e., significant improvement in general health, energy, balance, and better undisturbed sleep. In addition, it indicated reduction in joint pain, headaches, fatigue and digestive problems. The EOS data mirrors this with reports of improved breathing and feeling relaxed, stronger, and more energised (Suppl Figure S10). Diary entries of all participants in clusters 3 and 4 show improved physical wellbeing. Only six out of 59 participants reported no or minimal improvement. Our findings of physical improvement as a consequence of LSQ are consistent with the literature. A meta-analysis by [42] showed that Qigong exercise resulted in significantly improved physical ability compared with a control group. Qigong was found to enhance physical strength through the training of specific muscle groups [43], improve respiratory muscle strength [17], support immune system [44], and to reduce inflammation [45].

Data from the $B Q$ and the DE also suggests an improvement in the mental wellbeing of the participants. The analysis indicates significant reductions in stress, increased positivity, an enhanced ability to cope and improvement in emotional life. Consistently, the same benefits were reported in mental wellbeing: feeling calmer, positive, grounded, and peaceful (Suppl Figure S10). All participants in clusters 3 and 4 reported improved mental wellbeing. This is consistent with the findings from [46] stating that stress reduction and the regulation of emotion are characteristic benefits of Qigong.

The $B Q$ results show a significant reduction in loneliness and a greater connection to self and others. While the EOS survey shows benefits in relational wellbeing: improved connection to self and nature, being part of a bigger whole (Suppl Figure S10). All participants in clusters 3 and 4 consistently reported improved relational wellbeing. Our findings are similar to those of [47], [48] which emphasized individuals' strengthened sense of control, improved sense of social support and connection to others. Valence (kindness versus judgement and acceptance versus resentment) significantly changed over the course of the trial towards a kinder and more accepting attitude to self and others. The research literature supports a correlation between kindness, acceptance and positivity and wellbeing. Neff et al. and Gilbert suggest a link between 
kindness and compassion to self and others as a mediator for emotional wellbeing and happiness [49]-[52].

Furthermore, the EOS survey also demonstrates an improved sense of satisfaction and meaning, achievement, development of new knowledge, gratitude, and understanding. A better quality of life, self-care and health awareness were also observed. In the EOS survey, most of the participants $(45 / 63 \sim 71 \%)$ reported a significant improvement in overall wellbeing and positivity, and $64 \%$ of participants reported significant improvement in body awareness, awareness of mental activity and improved focus, awareness of Qi, awareness of connection to self and nature, and in satisfaction and meaning. The majority of the participants (64\%) in clusters 3 and 4 reported an increased sense of satisfaction and meaning, and all participants in clusters 3 and 4 reported improved awareness of body and focus. The BQ results also confirmed a significant increase in sensory and somatic awareness and in awareness of mental activity: feelings, thoughts, and images. Our findings are consistent with those of [53], which reported that self-connection predicts wellbeing and partially mediates the relationship between awareness and wellbeing. Focused awareness was demonstrated to improve brain function, mental activity, and interpersonal relationships [26], [54]-[60]. Self-awareness has been found to be consistently correlated with self-regulation [61].

Another interesting finding is that all participants who reported increased overall sense of satisfaction and meaning in the DEs and the EOS survey, also reported an increase in overall wellbeing as measured in the $\mathrm{BQ}$. There is strong evidence to suggest that meaning helps people establish better relationships, thus boosting relational and mental wellbeing [62]-[65]. Studies have found that people with a greater sense of meaning had lower toxic levels of stress, as marked by inflammatory cytokines and killer T-cell levels [66], [67].

Unexpectedly, participants with little or no prior experience of Qigong showed greater improvement in wellbeing than experienced practitioners (as stated in $\mathrm{H} 3$ ). It is possible that the experienced practitioners had already attained a high degree of wellbeing. These findings are consistent with our definition of wellbeing 'as the human experience of optimum harmonious functioning of body, mind, awareness, and relational processes'. Wellbeing emerges through the integrated functioning of these processes subservient to autopoiesis and nurturing life [28]. Findings in neurobiology suggest that interventions that include integration physical, mental, awareness and relational processes have a higher impact on wellbeing [30]-[33], [68]. We thus hypothesise that LSQ, by integrating physical relaxation, slow movement, and focused awareness of internal and external experience, contributes to all aspects of wellbeing.

Despite the strengths of the study and the contribution to existing research, there are certain limitations that need to be noted. The data collected was based on the self-evaluation and reflection of the participants rather than measuring physiological markers. We aim to further investigate the impacts of LSQ by integrating perceptual correlates of wellbeing with bio markers such as blood pressure, heart rate variability, respiration, and brain activity.

\section{Methods}

Participants joined live Qigong sessions over Zoom, once a week on Saturdays between 13:00 and 14:00 GMT over an eight-week period. The participants were asked to complete an online diary, as well as a questionnaire at three different time points (induction week, midpoint, and 
endpoint). Questionnaire responses were analysed to understand whether there were significant differences in scores across different timepoints. The online diary allowed participants to enter free-text data which could be completed multiple times during any given week immediately after Qigong practice. Free text data from the online diary was analysed using natural language processing (NLP) and clustering techniques. Diary text data was also evaluated with a thematic analysis tool known as NVivo.

A separate control group was recruited and asked to complete an identical questionnaire at the same three time-points during the pilot study.

\section{Recruitment to the Qigong practice and control groups}

A webpage describing the Qigong Research project was created on the organiser's website. The webpage included a registration form where volunteer participants could register their interest by entering their names, email addresses and indicating their level of experience of qigong practice. A similar webpage was created to recruit control group volunteers. In addition to email advertising sent to networks and mailing lists of people who had previously attended Chinese wellbeing workshops, a social media campaign was launched on Facebook and Twitter, directing interested individuals to the Qigong Research project webpage. The same process was followed to publicise the call for control group volunteers. An introductory online welcome event was hosted by the research team where potential volunteers listened to presentations on the project and were able to ask questions. Subsequently, the practice and control group volunteers were asked to confirm their participation through the web-based platform.

\section{Preparation of the screening tools and baseline questionnaire}

The Qigong Practice Research Project is a web-based platform that allows participants to follow the LSQ exercise and to maintain a practice diary noting their experiences. New users registered online (see Suppl Figure S11) to create an account ensuring secure logon and access to the application. Upon registration participants were able to access information on the study in order to consent to participation and comply with ethical approval procedures. Demographic data and participants' questionnaire information was anonymised.

After first logging in the participants completed a general health and wellbeing questionnaire (see Suppl Figure S3) to establish their personal baseline. Questions included, for example, general health, quality of sleep, stress (using a scale of 1-100) and whether participants had Covid-19 or long-Covid (yes / no responses). At the mid and endpoints of the trial the questionnaire was reactivated, and the participants asked to complete it again. Quantitative and qualitative data was collected to enable both statistical analysis and thematic and sentiment analysis of the baseline questionnaires to measure changes in health and the potential impact of the LSQ practice over the course of the trial.

\section{Practice videos and practice diary}

Once logged in participants were able to access a sequence of four videos demonstrating the practice routine of the LSQ exercise (Suppl Figure S12 and S13). These were recorded by an experienced TCM Qigong trainer who also delivered the weekly live-streamed sessions. 
Participants were asked to watch and practice the whole sequence at least once a week. The practice videos were hosted on YouTube and were embedded within the online platform (Suppl Figure S13), for ease of navigation.

Participants were asked to complete the practice diary (Suppl Figure S1) after each session. The diary approach [36]-[38] was adopted to implement an autopoietic perspective by creating the conditions for paying attention to and accessing one's immediate personal experience through a disciplined act of cultivating the capacity 'of becoming aware' of the sources of this experience [55]. The design of the diary reflects the five dimensions of the integrative wellbeing model shown in Figure 1 and allowed free articulation of experience. These included noting instances of body awareness (including changes to breathing after the practice), focused attention, Qi energy awareness, relational awareness (sense of connection to self and others) and sense of satisfaction and meaning. The data was analysed using thematic and sentiment analysis methods, as well as machine learning (natural language processing-NLP) techniques.

The Qigong practice platform also incorporated a 'My Diary' feature (Suppl Figure S14) which allows participants to manage personal account details and to view previous entries and baseline questionnaire responses.

\section{Quantitative analysis of the baseline questionnaire}

There are three separate scoring schemes for the baseline questionnaire items. The first is based on a scale of 1-100 (the lower the score, the better the improvement). The second is scaled 1-4 (the higher the score, the better the improvement), and the last one is a binary scoring scheme (yes / no response). The questionnaire items and scoring scheme are summarized in Suppl Table S2.

We investigated changes in the questionnaire scores across the three timepoints (the beginning, mid and endpoints of the study). Our analyses were based on both an integrative approach in which we combined scores from all the questionnaire items, and an individual approach in which we analysed each questionnaire item separately. The former analysis gave us an overview of the overall tendency in the score changes, while the latter provided us a finer grained analysis, and specific details regarding the score changes for each separate question. In our integrative analysis, questionnaire items scored between 1-100 were combined together (Figure 3A). Similarly, items scored between 1-4 were combined together (Figure 3B) and were separately analysed across the three timepoints. In the individual analysis, we tracked the score changes in each questionnaire item separately (e.g., score changes in general health were plotted, individually - Figure 4) across different time points.

We applied a non-parametric test - the Mann-Whitney-Wilcoxon (Wilcoxon rank sum) test - to compare the mean score of questionnaire items in each time point in a pairwise manner (i.e., initial timepoint versus midpoint [I2M] scores, midpoint versus endpoint [M2E], and initial versus endpoint [12E]). Additionally, we applied another non-parametric test - Kruskal-Wallis - for multiple group comparison to check whether there was any significant change in scores among all three timepoints. Both the non-parametric pairwise (Wilcoxon rank sum) and multiple group comparison (Kruskal-Wallis) tests were applied to the integrated and individual analysis of the questionnaire items. In addition to these comparisons across three timepoints within the practice and control groups, a comparison between the practice and control samples within each 
timepoint was also conducted using the Wilcoxon rank sum test. All analyses were performed in $R$. Boxplots demonstrating the scores of the questionnaire items were created in $\mathrm{R}$ using the ggboxplot function available in the ggplot library. In addition, to illustrate the mean scores of the questionnaire items in boxplots, we utilised a radar (spider) plot using the e radar function from the echarts $4 r$ library in R. Correlation scores between the pairs of questionnaire items were calculated with the Spearman coefficients.

\section{Semantic and thematic analyses of the diary entries}

The qualitative data were evaluated using thematic analysis. The data were uploaded and organised in NVIVO12 Software [69]. The analysis focused on discerning experiences within the physical, mental, relational and awareness domains as observed by the participants. The text data were interpreted twice, once by a researcher who did not have experience of Qigong and a second time by a researcher with extensive experience of Qigong, then discussed and agreed within the team. The data entries were manually clustered into 4 categories already established by the sentiment analysis: - cluster 0 : no change, cluster 1: low change, cluster 2: moderate change, cluster 3: high level of change, thus accounting for individual differences in receptiveness to the practice.

\section{Machine-learning-based automatic pattern recognition for classifying the impact of Qigong}

A natural language processing (NLP) approach and multiple Python libraries (including numpy, pandas and matplotlib [70]) were used to analyse the text data from the diary entries. The text was first pre-processed as follows: sentences were tokenized into words, all data was converted into lower case, words were lemmatized to find their roots, stopwords (non-informative but frequently repeated words such as the, $a$, of, and) were eliminated from the corpus by using the clean_doc function. Then the frequencies of the curated words were counted; term frequency (TF=[number of repetitions of a word in an entry] / [total number of words in an entry]) and inverse document frequency (IDF=Log[(total number of entries) / (number of entries containing this word)]) statistics of words were calculated and each word in each entry was represented by a TF*IDF score (a product of the TF and IDF statistics). Thereby, a numerical data matrix (TFIDF) in which each entry (1,265 in total) was used as an observation, and each informative word in the vocabulary was treated as an attribute $(3,403$ dimensions in total) was created from the text data. Then a heuristic k-means clustering approach was applied to identify the optimal number of clusters contained in the TF-IDF score matrix, each cluster representing a specific level of benefit derived from the LSQ practice. The search range of the optimal cluster number ( $k$ value) was [2, 9]. For each value of $k$, k-means clustering was implemented as follows: (i) randomly initialize the centroids of the $k$ clusters, (ii) for each data instance (diary entry), calculate its Euclidean distances to all the cluster centroids and assign the instance to the corresponding cluster based on its proximity to the centroids, (iii) after all the samples are assigned to the closest group, update the position of the centroids for each cluster by calculating the mean of all data instances affiliated to that cluster, (iv) repeat steps (ii) and (iii) until the solution is converged, or the maximum number of iterations is reached. Convergence means that the cluster centroids become stable (which means the label assignments of all 
samples no longer change). The optimal $k$ value was determined by using the elbow method from the yellowbrick library by calculating and plotting the 'distortion score' curve (the sum of square value of Euclidean distance between each point and the center of its assigned cluster) against the changing values of $k$ to point out the knee point of this curve (Suppl Figure S8). The knee point on the curve corresponds to the optimal $k$ which minimizes the within cluster variations.

$$
J=\sum_{i}^{n} \sum_{O_{j} \in C_{i}}\left(O_{j}-Z_{i}\right)^{2}
$$

where $i$ and $Z_{i}$ represent the $i$ th cluster and its centroid. Besides, $O_{j}$ and $n$ represent the data samples belonging to the $i$ th cluster and the total number of clusters, respectively.

After the identification of the optimal value of $k$, we searched for the proper text-based tags for each numerical cluster label. The top 100 most frequent words of each cluster were listed (Suppl Table S3) and were compared to the word sets derived from the NVivo tool classifications. The most similar NVivo-driven word sets, and k-means-driven word sets were hypothesized to represent the same pattern, indicating that the NVivo-driven text label could be used for tagging the numerical label of the corresponding $k$-means group. Additionally, sentiment analysis was used to identify the cluster labels. Firstly, the top 100 words for each cluster were collected and cleaned by removing duplication. Then, the python library textblob was applied to these four groups of words. Finally, the sentiment scores within the range of [-1 1] were calculated for the purpose of evaluating the level of benefit represented by each cluster, i.e., the higher the sentiment score, the higher the positive impact from the LSQ practice.

\section{End of study (EOS) survey evaluations and comparison between EOS and other data resources}

The end of study (EOS) survey allowed the participants to reflect their overall satisfaction level, share their insights, and to score their experiences of (i) body, (ii) Qi, (iii) focused awareness, (iv) connection to others and nature, (v) satisfaction, and (vi) positive wellbeing. The closed questions (i)-(vi) in the EOS survey scored between 1-100. The positivity of the experience and satisfaction level increases as the score rises. The maximum total scores from the evaluation of the closed questions can be as high as 600 .

EOS survey results were compared to those of the baseline questionnaires as well as the diary entries. We hypothesized that, if a participant was satisfied with the study, then they would award a score of at least $65 \%$ for each question in EOS, resulting in a score of 390 for all 6 questions, rounded up to 400 . If the majority of the diary entries from a participant belonged to clusters 3 and 4 (moderate and high level of change) and were followed by an EOS score of more than 400 , then that participant was considered to have a highly consistent positive experience throughout the entire journey of LSQ practice. We labeled such users as Pseudo True Positive (PTP) samples. If a participant's entries fell in clusters 1 or 2 (no or low-level change) but the EOS score was above the (default) value of $50 \%$ per question (>300 in total), we considered them as Pseudo False Negatives (PFN) since the EOS directly reflects the satisfaction level (clearly and manually determined by the participants), whereas the cluster labels from the diary entries are data-driven and predicted automatically. If the diary entries 
reflected a low level of satisfaction (clusters 1 or 2), the EOS was also expected to be low (at least lower than average); if the EOS was higher than average, then it was supposed to be a misclassified (PFN) sample. Hence, while defining the PFN, we used a stringent threshold (>300), rather than a smaller value to identify the misclassified samples. This meant we penalized ourselves with a high level of PFN in order to achieve a more robust model. If both the diary-based clustering and EOS results consistently indicated no change, or a low-level of satisfaction or change (clusters 1 or 2 and $E O S<200$ ), then this group of practitioners were classified as the Pseudo True Negatives (PTN). Lastly, if the participant experienced a relatively high level of change (clusters 3 or 4), while giving low scores for the EOS survey $(<200)$ they were labeled as a Pseudo False Positive (PFP, see Table 2).

\section{References}

[1] S. Hu and C. Duan, "Effect of Qigong on the prevention and treatment of respiratory tract infection in the Elderly," East. Qigong, vol. 4, pp. 32-33, 1992.

[2] W. PA, I. KE, A. J, B. VE, and O. JE, "A pilot study of qigong practice and upper respiratory illness in elite swimmers," Am. J. Chin. Med., vol. 39, no. 3, pp. 461-475, 2011, doi: 10.1142/S0192415X11008968.

[3] C. K and Y. R, "Exploratory studies of Qigong therapy for cancer in China," Integr. Cancer Ther., vol. 1, no. 4, pp. 345-370, Dec. 2002, doi: 10.1177/1534735402238187.

[4] S. KM, "Therapeutic benefits of qigong exercises in combination with drugs," J. Altern. Complement. Med., vol. 5, no. 4, pp. 383-389, 1999, doi: 10.1089/ACM.1999.5.383.

[5] J. Fu, S. Fu, and J. Qin, "Effect of qigong and anticancer body-building herbs on the prognosis of postoperative patients with cardiac adenocarcinoma," in Third World Conference on Medical Qigong, 1996.

[6] E. Hong, "The effect of Yudongkong exercise in fatigue, difficulty of daily activities and symptoms of side effect in advanced gastric cancer patients receiving chemotherapy.," Yonsei University, Seoul, Korea, 2003.

[7] S. Lam, "A randomized, controlled trial of Guolin qigong in patients receiving tanscatheter arterial chemoembolisation for unresectable hepatocellular carcinoma," University of Hong Kong, Hong Kong, 2004.

[8] L. TI, C. HH, and Y. ML, "Effects of chan-chuang qigong on improving symptom and psychological distress in chemotherapy patients," Am. J. Chin. Med., vol. 34, no. 1, pp. 37-46, 2006, doi: 10.1142/S0192415X06003618.

[9] J. Manzaneque et al., "Assessment of immunological parameters following a qigong training program - PubMed," Med. Sci. Monit., vol. 10, no. 6, 2004.

[10] L. MS et al., "Effects of Qigong on immune cells," Am. J. Chin. Med., vol. 31, no. 2, pp. 327-335, 2003, doi: 10.1142/S0192415X03001016. 
[11] L. MS, L. MS, K. HJ, and C. ES, "Effects of qigong on blood pressure, high-density lipoprotein cholesterol and other lipid levels in essential hypertension patients," Int. J. Neurosci., vol. 114, no. 7, pp. 777-786, Jul. 2004, doi: 10.1080/00207450490441028.

[12] C. BM et al., "Randomised controlled trial of qigong in the treatment of mild essential hypertension," J. Hum. Hypertens., vol. 19, no. 9, pp. 697-704, Sep. 2005, doi: 10.1038/SJ.JHH.1001884.

[13] C. SHW and T. HWH, "The beneficial effects of Qigong on elderly depression," Int. Rev. Neurobiol., vol. 147, pp. 155-188, Jan. 2019, doi: 10.1016/BS.IRN.2019.06.004.

[14] W. W. Y. So, S. Cai, S. Y. Yau, and H. W. H. Tsang, "The Neurophysiological and Psychological Mechanisms of Qigong as a Treatment for Depression: A Systematic Review and Meta-Analysis," Front. Psychiatry, vol. 10, p. 820, Nov. 2019, doi: 10.3389/FPSYT.2019.00820.

[15] T. HW and F. KM, "A review on neurobiological and psychological mechanisms underlying the anti-depressive effect of qigong exercise," J. Health Psychol., vol. 13, no. 7, pp. 857-863, Oct. 2008, doi: 10.1177/1359105308095057.

[16] F. Wang et al., "The Effects of Qigong on Anxiety, Depression, and Psychological WellBeing: A Systematic Review and Meta-Analysis," Evid. Based. Complement. Alternat. Med., vol. 2013, p. 16, 2013, doi: 10.1155/2013/152738.

[17] W. Wu et al., "Effects of qigong on late-stage complex regional pain syndrome.," Altern Ther Heal. Med., vol. 5, no. 1, pp. 45-54, 1999.

[18] M. S. LEE, C.-W. KANG, and H. RYU, "Acute Effect of Qi-Training on Natural Killer Cell Subsets and Cytotoxic Activity," https://doi.org/10.1080/00207450590519580, vol. 115, no. 2, pp. 285-297, Feb. 2009, doi: 10.1080/00207450590519580.

[19] L. MS, L. HJ, and L. MS, "Impact of qigong exercise on self-efficacy and other cognitive perceptual variables in patients with essential hypertension," J. Altern. Complement. Med., vol. 10, no. 4, pp. 675-680, Aug. 2004, doi: 10.1089/ACM.2004.10.675.

[20] M. K and A. M, "Efficacy and feasibility of a combination of body awareness therapy and qigong in patients with fibromyalgia: a pilot study," J. Rehabil. Med., vol. 36, no. 6, pp. 279-281, Nov. 2004, doi: 10.1080/16501970410031912.

[21] T. Tsujiuchi et al., "The Effect of Qi-Gong Relaxation Exercise on the Control of Type 2 Diabetes Mellitus," Diabetes Care, vol. 25, no. 1, pp. 241-242, Jan. 2002, doi: 10.2337/DIACARE.25.1.241.

[22] O. B et al., "The Effects of Tai Chi and Qigong on Immune Responses: A Systematic Review and Meta-Analysis," Med. (Basel, Switzerland), vol. 7, no. 7, p. 39, Jun. 2020, doi: 10.3390/MEDICINES7070039.

[23] S.-H. Hung, C.-Y. Hwang, and C.-Y. Chang, "Is the Qi experience related to the flow experience? Practicing qigong in urban green spaces," PLoS One, vol. 16, no. 1, p. e0240180, Jan. 2021, doi: 10.1371/JOURNAL.PONE.0240180. 
[24] N. BH and T. HW, "Psychophysiological outcomes of health qigong for chronic conditions: a systematic review," Psychophysiology, vol. 46, no. 2, pp. 257-269, Mar. 2009, doi: 10.1111/J.1469-8986.2008.00763.X.

[25] P. Sice et al., "An autopoietic approach to assessing the impact of TCM Qigong exercise on wellbeing: A feasibility study," Systemist, vol. 41, no. 1, pp. 108-132.

[26] P. Sice, G. Elvin, C. Riachy, Y. Shang, S. Ogwu, and C. Zink, "Online screening of Xsystem music playlists using an integrative wellbeing model informed by the theory of autopoiesis," IEEE Access, vol. 8, pp. 182307-182319, 2020, doi: 10.1109/ACCESS.2020.3029142.

[27] H. R. Maturana and G. Verden-Zöller, The Origin of Humanness in the Biology of Love . Imprint Academic, 2012.

[28] P. Sice and I. French, "Understanding Humans and Organisations: Philosophical Implications of Autopoiesis," Philos. Manag., vol. 4, no. 1, pp. 55-66, Mar. 2004, doi: 10.5840/POM20044114.

[29] F. Varela, "Neurophenomenology: A methodological remedy for the hard problem," J. Conscious. Stud., vol. 3, no. 4, 1996.

[30] F. J. Varela, "Patterns of Life: Intertwining Identity and Cognition," Brain Cogn., vol. 34, no. 1, pp. 72-87, Jun. 1997, doi: 10.1006/BRCG.1997.0907.

[31] S. W. Porges, The Polyvagal Theory: Neurophysiological Foundations of Emotions, Attachment, Communication, and Self-Regulation (Audio Download): Stephen W. Porges, Joe Hempel, Tantor Audio: Amazon.co.uk: Books. New York: W. W. Norton \& Company, 2011.

[32] A. R. Damásio, The Strange Order of Things: Life, Feeling, and the Making of Cultures. Pantheon, 2018.

[33] F. Varela, J.-P. Lachaux, E. Rodriguez, and J. Martinerie, "The brainweb: Phase synchronization and large-scale integration," Nat. Rev. Neurosci. 2001 24, vol. 2, no. 4 , pp. 229-239, 2001, doi: 10.1038/35067550.

[34] G. Deco, G. Tononi, M. Boly, and M. L. Kringelbach, "Rethinking segregation and integration: contributions of whole-brain modelling," Nat. Rev. Neurosci. 2015 167, vol. 16, no. 7, pp. 430-439, Jun. 2015, doi: 10.1038/nrn3963.

[35] C. Zhang, F. A. Popp, and M. Bischof, Current development of biophysics : the stage from an ugly duckling to a beautiful swan, 1st ed. [Hangzhou]: Hangzhou University Press, 1996.

[36] R. Bartlett and C. Milligan, What is Diary Method? Bloomsbury Academic, 2015.

[37] D. McDuff, A. Karlson, A. Kapoor, A. Roseway, and M. Czerwinski, "AffectAura: Emotional wellbeing reflection system," in 2012 6th International Conference on Pervasive Computing Technologies for Healthcare and Workshops, PervasiveHealth 2012, 2012, pp. 199-200, doi: 10.4108/icst.pervasivehealth.2012.248727. 
[38] D. Pavel, V. Callaghan, and A. K. Dey, "From self-monitoring to self-understanding: Going beyond physiological sensing for supporting wellbeing," in 2011 5th International Conference on Pervasive Computing Technologies for Healthcare and Workshops, PervasiveHealth 2011, 2011, pp. 312-315, doi: 10.4108/ICST.PERVASIVEHEALTH.2011.245999.

[39] J. Uher, "Quantitative data from rating scales: An epistemological and methodological enquiry," Front. Psychol., vol. 9, no. DEC, Dec. 2018, doi: 10.3389/FPSYG.2018.02599/FULL.

[40] L. Tay and A. T. Jebb, "Establishing Construct Continua in Construct Validation: The Process of Continuum Specification," Adv. Methods Pract. Psychol. Sci., vol. 1, no. 3, pp. 375-388, Sep. 2018, doi: 10.1177/2515245918775707.

[41] J. D. Abbey and M. G. Meloy, "Attention by design: Using attention checks to detect inattentive respondents and improve data quality," J. Oper. Manag., vol. 53-56, pp. 6370, Nov. 2017, doi: 10.1016/J.JOM.2017.06.001.

[42] C. PS, K. T, O. B, and F. M, "Physical and Psychological Health Outcomes of Qigong Exercise in Older Adults: A Systematic Review and Meta-Analysis," Am. J. Chin. Med., vol. 47, no. 2, pp. 301-322, 2019, doi: 10.1142/S0192415X19500149.

[43] Y. Liu and S. Hu, "The Effect of Qigong on muscle function of older adults," J Gerontol., vol. 4, no. 228, 1988.

[44] C. J et al., "Tai Chi Chuan increases circulating myeloid dendritic cells," Immunol. Invest., vol. 39, no. 8, pp. 863-873, 2010, doi: 10.3109/08820139.2010.503766.

[45] I. MR et al., "Cognitive behavioral therapy and tai chi reverse cellular and genomic markers of inflammation in late-life insomnia: a randomized controlled trial," Biol.

Psychiatry, vol. 78, no. 10, pp. 721-729, Nov. 2015, doi:

10.1016/J.BIOPSYCH.2015.01.010.

[46] F. Feng, S. Tuchman, J. W. Denninger, G. L. Fricchione, and A. Yeung, "Qigong for the Prevention, Treatment, and Rehabilitation of COVID-19 Infection in Older Adults," Am. J. Geriatr. Psychiatry, vol. 28, no. 8, pp. 812-819, Aug. 2020, doi: 10.1016/J.JAGP.2020.05.012.

[47] J. Y. man Siu, "Coping with future epidemics: Tai chi practice as an overcoming strategy used by survivors of severe acute respiratory syndrome (SARS) in post-SARS Hong Kong," Heal. Expect., vol. 19, no. 3, pp. 762-772, Jun. 2016, doi: 10.1111/HEX.12270.

[48] S. JY, S. HC, and L. WL, "Qigong practice among chronically ill patients during the SARS outbreak," J. Clin. Nurs., vol. 16, no. 4, pp. 769-776, Apr. 2007, doi: 10.1111/J.13652702.2006.01695.X.

[49] K. D. Neff and A. P. Costigan, "Self-Compassion, Wellbeing , and Happiness," Psychol. Österreich, vol. 2, no. 3, pp. 114-119, 2014.

[50] N. KD and G. CK, "A pilot study and randomized controlled trial of the mindful selfcompassion program,” J. Clin. Psychol., vol. 69, no. 1, pp. 28-44, Jan. 2013, doi: 
10.1002/JCLP.21923.

[51] P. Gilbert, Compassion : concepts, research and applications. London: Routledge, 2017.

[52] P. Gilbert, The Compassionate Mind: A New Approach to Life's Challenges. New Harbinger Publications, 2010.

[53] K. Klussman, N. Curtin, J. Langer, and A. L. Nichols, "Examining the effect of mindfulness on well-being: self-connection as a mediator:," https://doi.org/10.1017/prp.2019.29, vol. 14, p. 5, Jan. 2020, doi: 10.1017/PRP.2019.29.

[54] M. E. Martinez, "The Process of Knowing: A Biocognitive Epistemology," J. Mind Behav., pp. 407-426, 2001.

[55] N. Depraz, F. J. Varela, and P. Vermersch, On Becoming Aware. A pragmatics of experiencing. John Benjamins Publishing Company, 2003.

[56] R. R. Llinás, "Of Self and Self Awareness The Basic Neuronal Circuit in Human Consciousness and the Generation of Self," J. Conscious. Stud., vol. 9, pp. 64-74, 2008.

[57] K. S. Cohen, The Way of Qi Gong: The Art and Science Energy Healing. Wellspring/Ballantine, 2018.

[58] D. J. Siegel, "Mindful Awareness, Mindsight, and Neural Integration," J. Humanist. Psychol., vol. 37, no. 2, pp. 137-158, 2009.

[59] D. J. Siegel, "An interpersonal neurobiology approach to psychotherapy: Awareness, mirror neurons, and neural plasticity in the development of well-being," Psychiatr. Ann., vol. 36, no. 4, pp. 248-256, 2006.

[60] P. Sice, E. Bentley, and L. Rauch, "Ontology, epistemology and the complexity of human neurobiology," Hum. Syst. Manag., vol. 37, no. 3, pp. 353-360, Jan. 2018, doi: 10.3233/HSM-171795.

[61] P. Verhaeghen and G. Mirabito, "When you are talking to yourself, is anybody listening? The relationship between inner speech, self-awareness, wellbeing, and multiple aspects of self-regulation," Int. J. Personal. Psychol., vol. 7, pp. 8-24, Aug. 2021, doi: 10.21827/IJPP.7.37354.

[62] M. F. Steger, Meaning and well-being. Handbook of well-being. Salt Lake City, UT, USA: DEF Publishers, 2018.

[63] T. F. Stillman, N. M. Lambert, F. D. Fincham, and R. F. Baumeister, "Meaning as Magnetic Force: Evidence That Meaning in Life Promotes Interpersonal Appeal," http://dx.doi.org/10.1177/1948550610378382, vol. 2, no. 1, pp. 13-20, Aug. 2010, doi: $10.1177 / 1948550610378382$.

[64] F. Martela, R. M. Ryan, and M. F. Steger, "Meaningfulness as Satisfaction of Autonomy, Competence, Relatedness, and Beneficence: Comparing the Four Satisfactions and Positive Affect as Predictors of Meaning in Life," J. Happiness Stud. 2017 195, vol. 19, no. 5, pp. 1261-1282, Mar. 2017, doi: 10.1007/S10902-017-9869-7. 
[65] F. Martela and K. M. Sheldon, "Clarifying the Concept of Well-Being: Psychological Need Satisfaction as the Common Core Connecting Eudaimonic and Subjective Well-Being:," https://doi.org/10.1177/1089268019880886, vol. 23, no. 4, pp. 458-474, Oct. 2019, doi: 10.1177/1089268019880886.

[66] B. JE, K. ME, T. SE, and F. JL, "Finding positive meaning and its association with natural killer cell cytotoxicity among participants in a bereavement-related disclosure intervention," Ann. Behav. Med., vol. 25, no. 2, pp. 146-155, 2003, doi: 10.1207/S15324796ABM2502_11.

[67] N. Krause and R. David Hayward, "Religion, Meaning in Life, and Change in Physical Functioning During Late Adulthood," J. Adult Dev. 2012 193, vol. 19, no. 3, pp. 158-169, Feb. 2012, doi: 10.1007/S10804-012-9143-5.

[68] F. J. Varela and J. Shear, The View from Within: First-Person Approaches to the Study of Consciousness, no. 5048. Imprint Academic, 1999.

[69] V. Braun and V. Clarke, "Using thematic analysis in psychology," Qual. Res. Psychol., vol. 3, no. 2, pp. 77-101, 2006, doi: 10.1191/1478088706QP063OA.

[70] P. Virtanen et al., "SciPy 1.0: Fundamental Algorithms for Scientific Computing in Python," Nat. Methods, vol. 17, no. 3, pp. 261-272, 2020.

\section{Acknowledgements}

We would like to specially acknowledge Prof Zhang Wenchun from Jiangxi University of Chinese Medicine and his work designing the Lung Strengthening Qigong Exercise, and his PhD student Zhao Zhangyang for her help as an instructor leading the live-streamed Qigong sessions. We would like to also thank Prof Zhao Yanxia from the University of Wales Trinity Saint David and Liu Yu from Beijing Union University for their support, also our colleagues Prof Wai Lok Woo, Dr John Arthur, Dr Katherine Butler, Dr Sam Smith, and Dr Inga Freimane from the University of Northumbria, Newcastle Upon Tyne, UK.

\section{Author contributions}

PS, KK, and ZK conceived the study, with PS responsible for the design of the experimental components, qualitative analysis, KK recruiting participants, arranging live-streamed Qigong sessions and ZK responsible for the design of the statistical and machine learning-based analysis, integration of the data streams. PS led the preparation of the baseline questionnaire (BQ), online diary, end of study survey (EOS) questions, qualitative analysis, SO ran the Nvivobased qualitative analysis on diary entries (DE). GE designed all of the online screening tools for collecting all different types of data. PS, KK, SO manually read, analyzed and categorized the online DEs from the participants, manually checked all text entries against data-driven clusters predicted by a machine learning approach. ZK and PW ran the statistical analysis on $\mathrm{BQ}$ and EOS. ZK, HX, PW designed and implemented the machine learning-based clustering of the DEs. SST helped revising the results from statistical data analysis and preparation of 
illustrations. ZK, PS, KK, GE drafted the manuscript, and all authors read, revised, and approved the final manuscript.

\section{Competing interests}

The authors declare no competing interests.

\section{Main Figures and Tables}

Table 1 - The centroid point and number of diary entries assigned to each cluster

\begin{tabular}{|l|l|l|l|l|l|l|l|}
\hline Cluster label & $\begin{array}{l}\text { Body } \\
\text { awareness }\end{array}$ & $\begin{array}{l}\text { Focused } \\
\text { attention }\end{array}$ & $\begin{array}{l}\text { Qi energy } \\
\text { awareness }\end{array}$ & $\begin{array}{l}\text { Relational } \\
\text { awareness }\end{array}$ & $\begin{array}{l}\text { Satisfaction } \\
\text { meaning }\end{array}$ & $\begin{array}{l}\text { Sentiment } \\
\text { score } \\
\text { (positivity) }\end{array}$ & $\begin{array}{l}\text { \# of entries in } \\
\text { this cluster }\end{array}$ \\
\hline $\mathbf{1}$ - No change & 0.167 & 0.175 & 0.034 & 0.185 & 0.447 & 0.153 & 296 \\
\hline $\begin{array}{l}\mathbf{2}-\text { Low-level of } \\
\text { change }\end{array}$ & 0.172 & 0.207 & 0.448 & 0.250 & 0.263 & 0.186 & 87 \\
\hline $\begin{array}{l}\mathbf{3}-\text { Medium-level } \\
\text { of change }\end{array}$ & 0.205 & 0.296 & 0.045 & 0.101 & 0.00076811 & 0.205 & 773 \\
\hline $\begin{array}{l}\mathbf{4}-\text { High-level of } \\
\text { change }\end{array}$ & 0.0373 & -0.031 & 0.030 & 0.039 & 0.017 & 0.229 & 199 \\
\hline \multicolumn{7}{|l|}{ Total number of entries } \\
\hline
\end{tabular}

Table 2 - Confusion matrix of the integrative evaluation of diary, baseline questionnaires, and EOS survey

\begin{tabular}{|l|l|l|l|}
\hline & $\begin{array}{l}\text { Participants whose EOS } \\
\text { survey scores }>=400\end{array}$ & $\begin{array}{l}\text { Participants whose EOS } \\
\text { survey scores }<=200\end{array}$ & $\begin{array}{l}\text { Total number of the } \\
\text { participants }\end{array}$ \\
\hline $\begin{array}{l}\text { Participants whose diary entries } \\
\text { were clustered in }\{3,4\} \text { [reflecting } \\
\text { a high-level positive change] and } \\
\text { have an improvement in baseline } \\
\text { questionnaires }\end{array}$ & $\begin{array}{l}\text { Pseudo True Positive } \\
\text { (PTP): } \\
28 / 44=64 \%\end{array}$ & $\begin{array}{l}\text { Pseudo False Positive } \\
\text { (PFP): } \\
6 / 44=14 \%\end{array}$ & $\begin{array}{l}\text { 59; but, } \\
\text { 44 among 59 (75\%) } \\
\text { were classified as in } \\
\text { [PTP, PTN, PFP, PFN }\} \\
\text { Labels of the rest (15 } \\
\text { users) were omitted (NA } \\
\text { value) }\end{array}$ \\
\hline $\begin{array}{l}\text { Participants whose diary entries } \\
\text { were clustered in }\{1,2\} \text { [reflecting } \\
\text { no or a low-level change] and } \\
\text { have stability [no improvement] } \\
\text { in baseline questionnaires }\end{array}$ & $\begin{array}{l}\text { [EOS scores }>=300] \\
\text { Pseudo False Negative } \\
\text { (PFN): } \\
5 / 44=11 \%\end{array}$ & $\begin{array}{l}\text { Pseudo True Negative } \\
\text { (PTN): } \\
5 / 44=11 \%\end{array}$ & \\
\hline
\end{tabular}




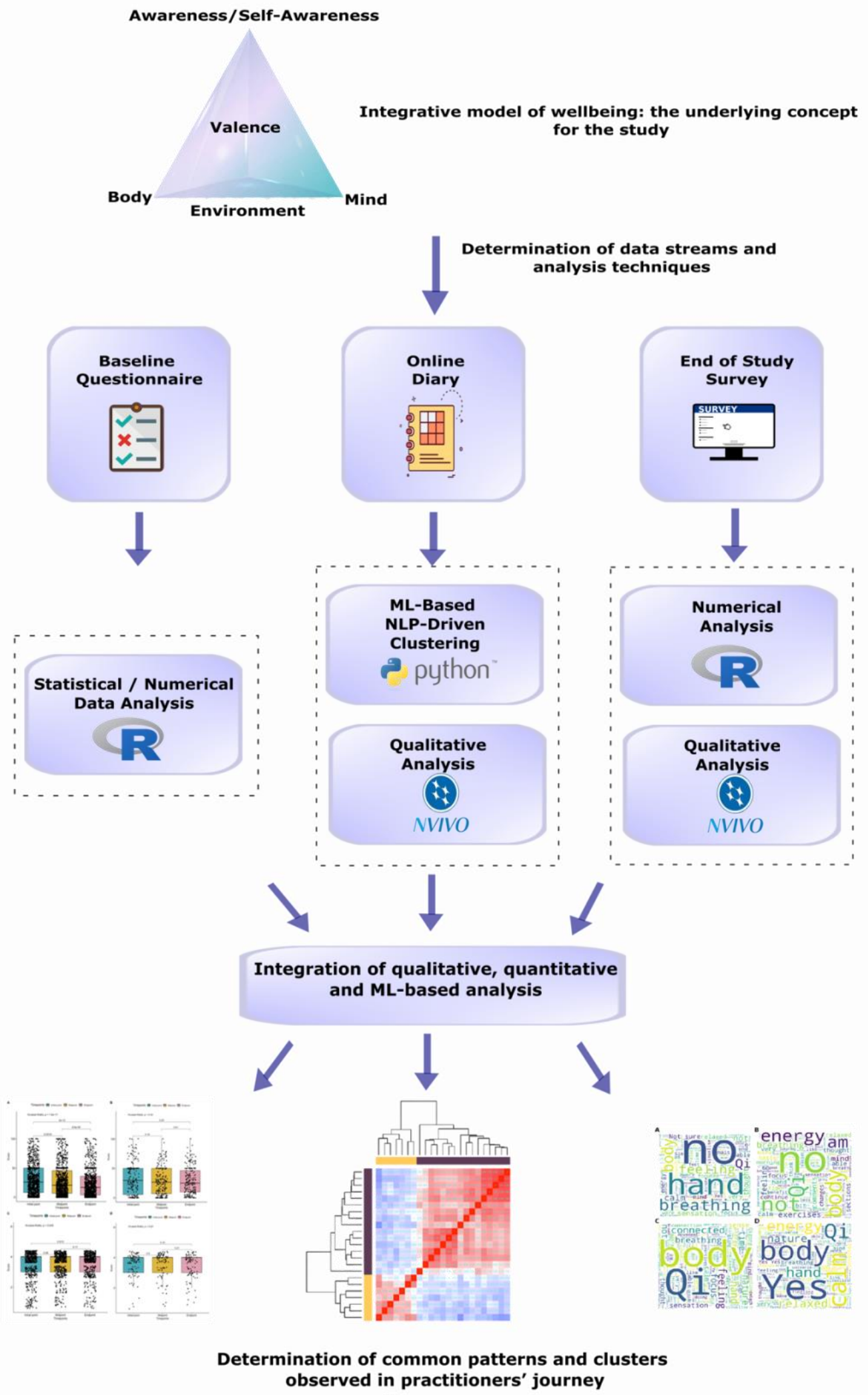

Figure 1 - Framework and underlying concept of the proposed study model 

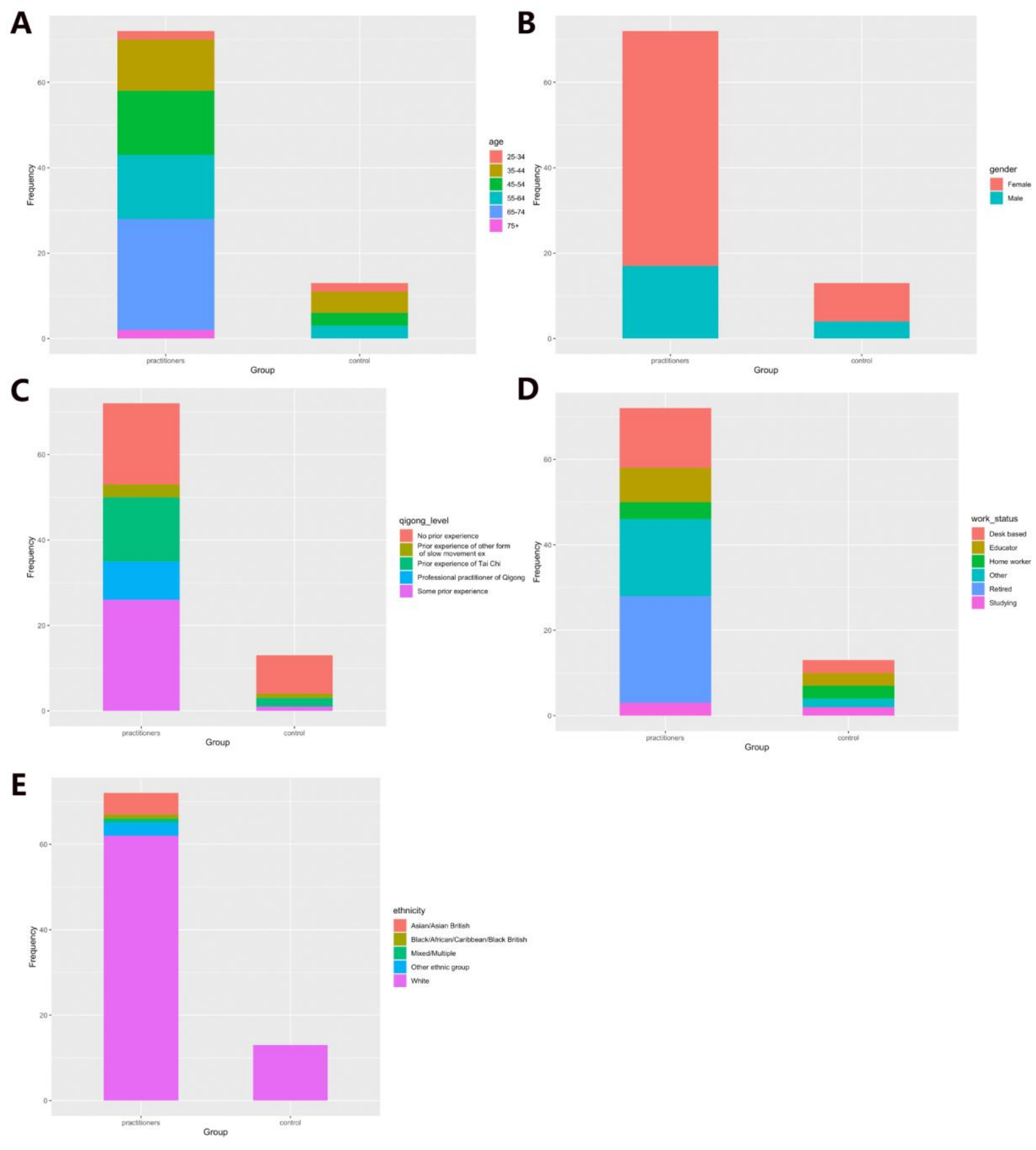

Figure 2 - Distribution of demographics based on (A) age, (B) gender, (C) previous experience with Qigong, (D) Occupation/work status, (E) Ethnicity 

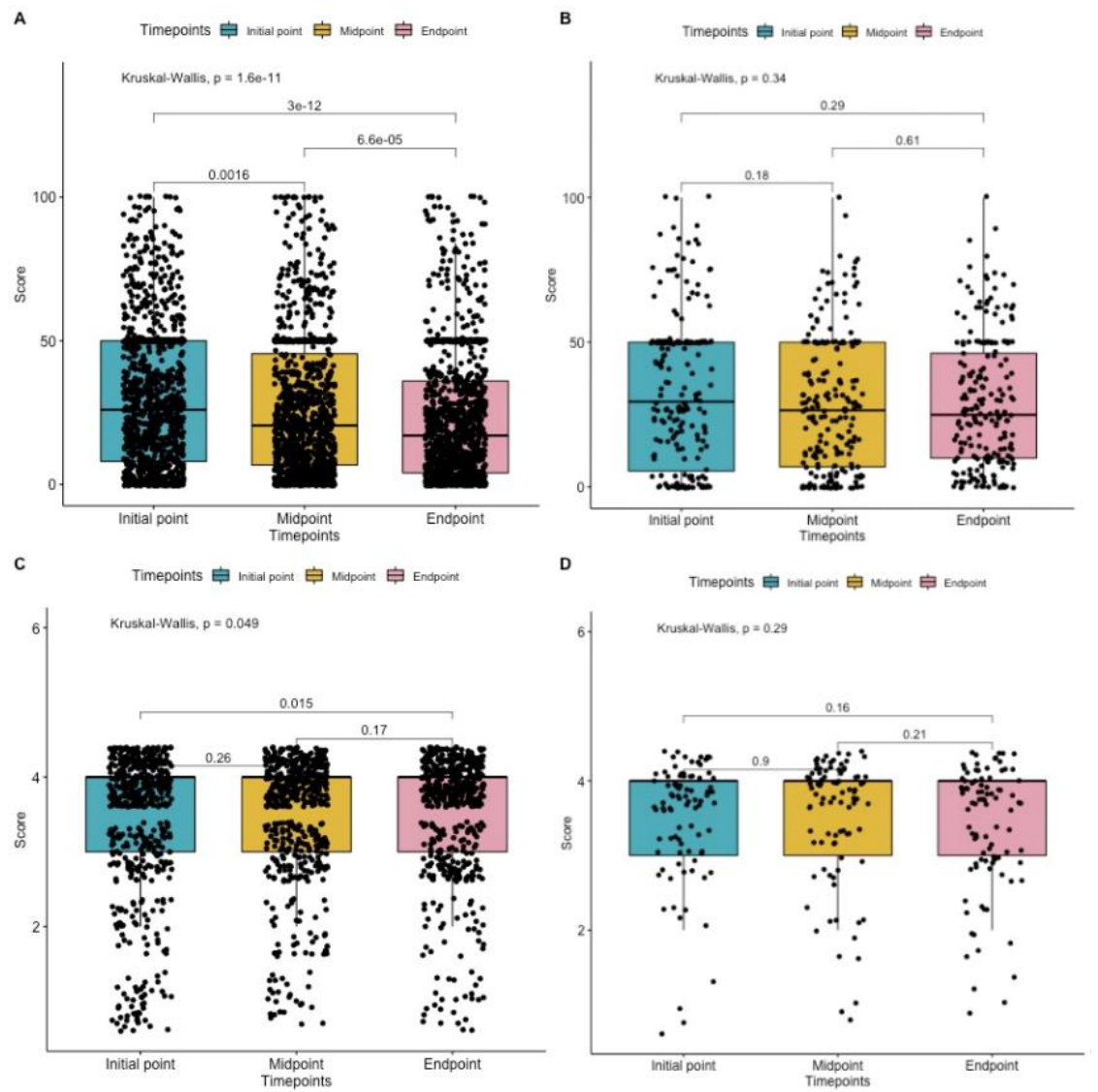

Figure 3 - Statistical analysis of $(A)$ items scored between 1-100 over 3 timepoints in practitioners group; (B) items scored between 1-100 in control group; (C) items scored 1-4 over 3 timepoints in practitioners group; (D) items scored 1-4 over 3 timepoints in control group 


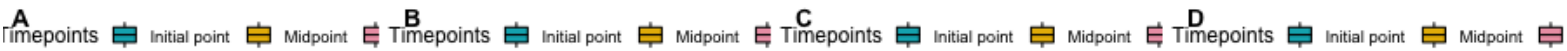

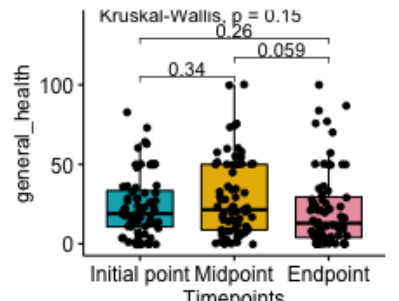

Timepoints

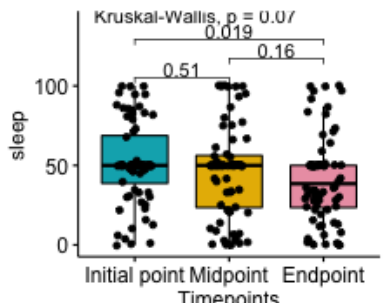

Timepoints

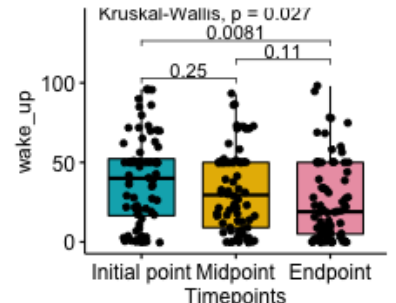

Timepoints

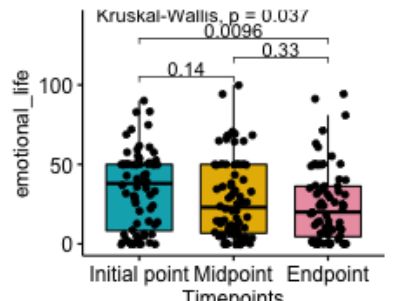

Timepoints

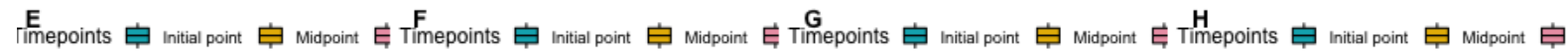

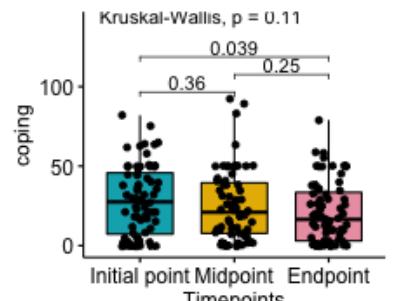

Timepoints

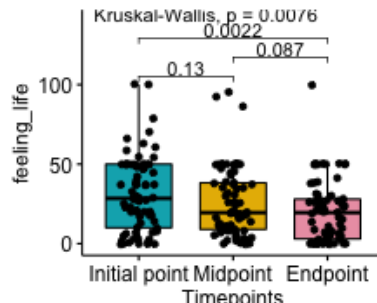

Timepoints

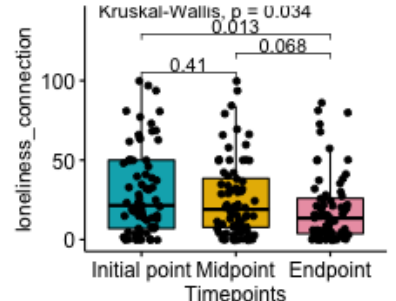

Timepoints

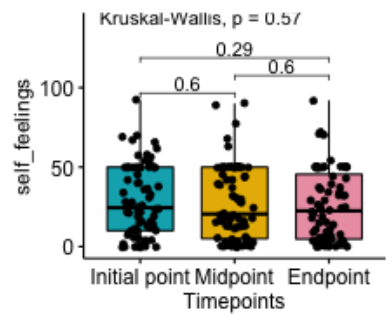

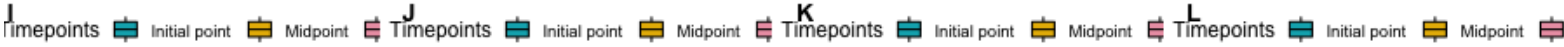
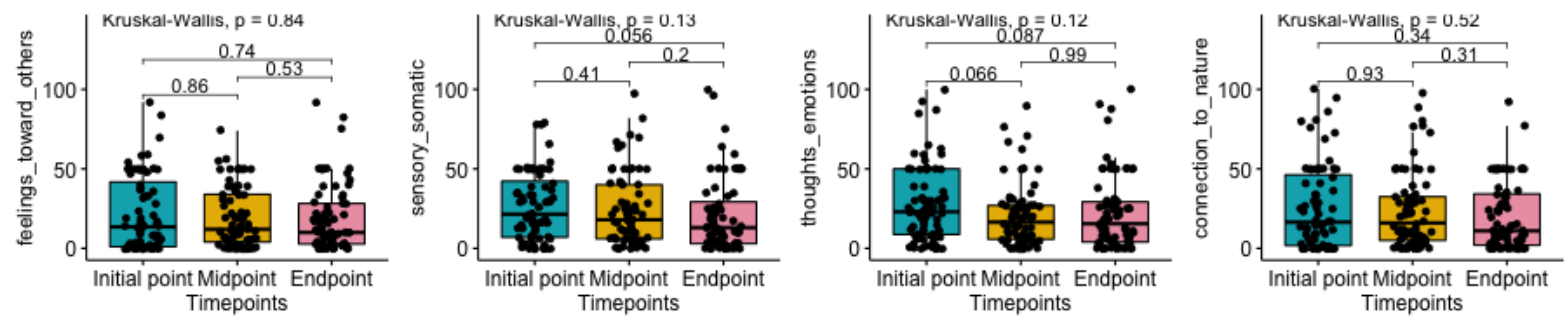

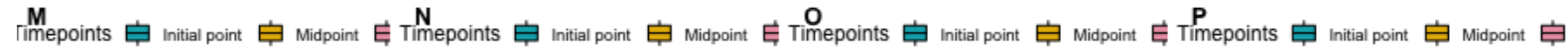
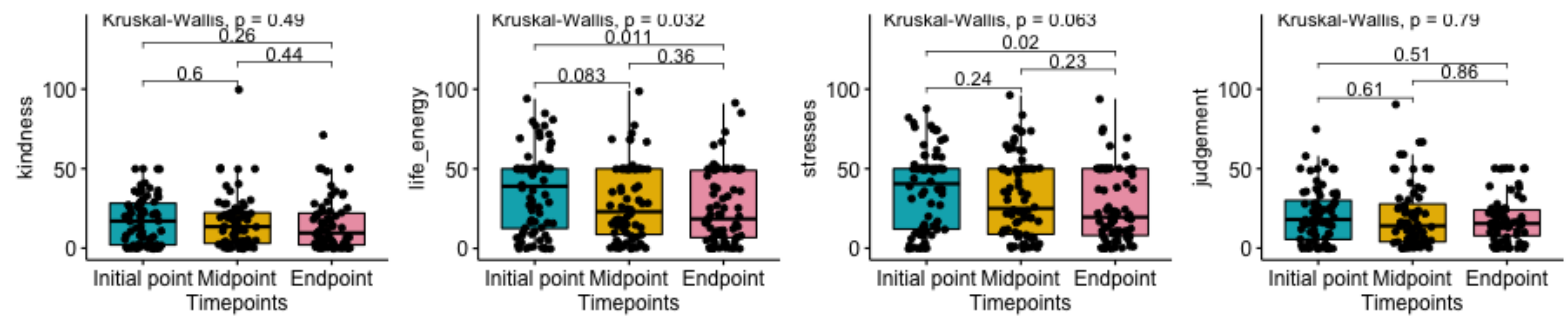

Figure 4A-P Individual analyses of the questionnaire items scored between 1-100 on practitioner group 
Timepoints 追 Initial point 追 Midpoint 追 Endpoir $\mathbf{S}$

S Timepoints 白 Initial point 白 Midpoint 白 Endpoir ${ }^{\top}$

Timepoints 追 Initial point 白 Midpoint 白 Endpoit
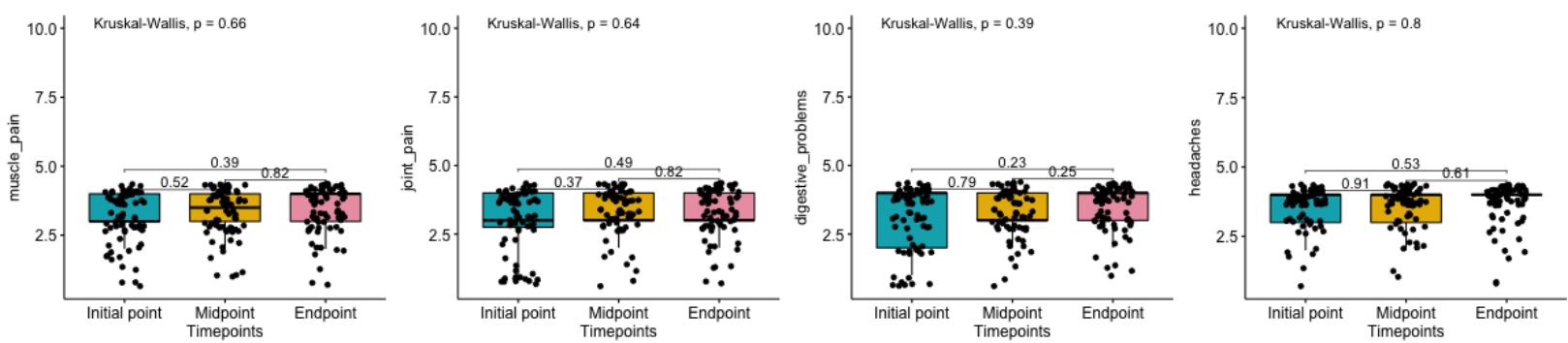

U Timepoints 白 Initial point 白 Midpoint 白 Endpoir
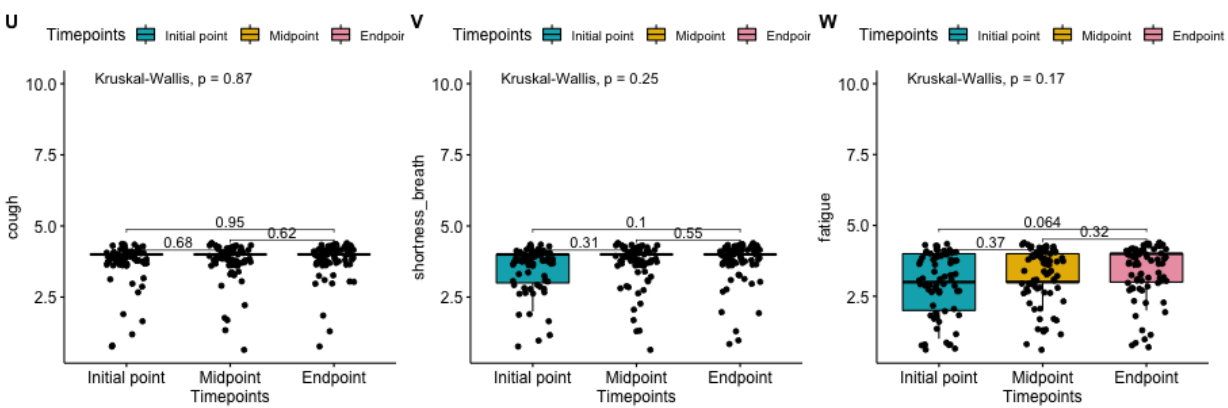

Figure 4Q-W Individual analyses of the questionnaire items scored between 1-4 on practitioner group 
A

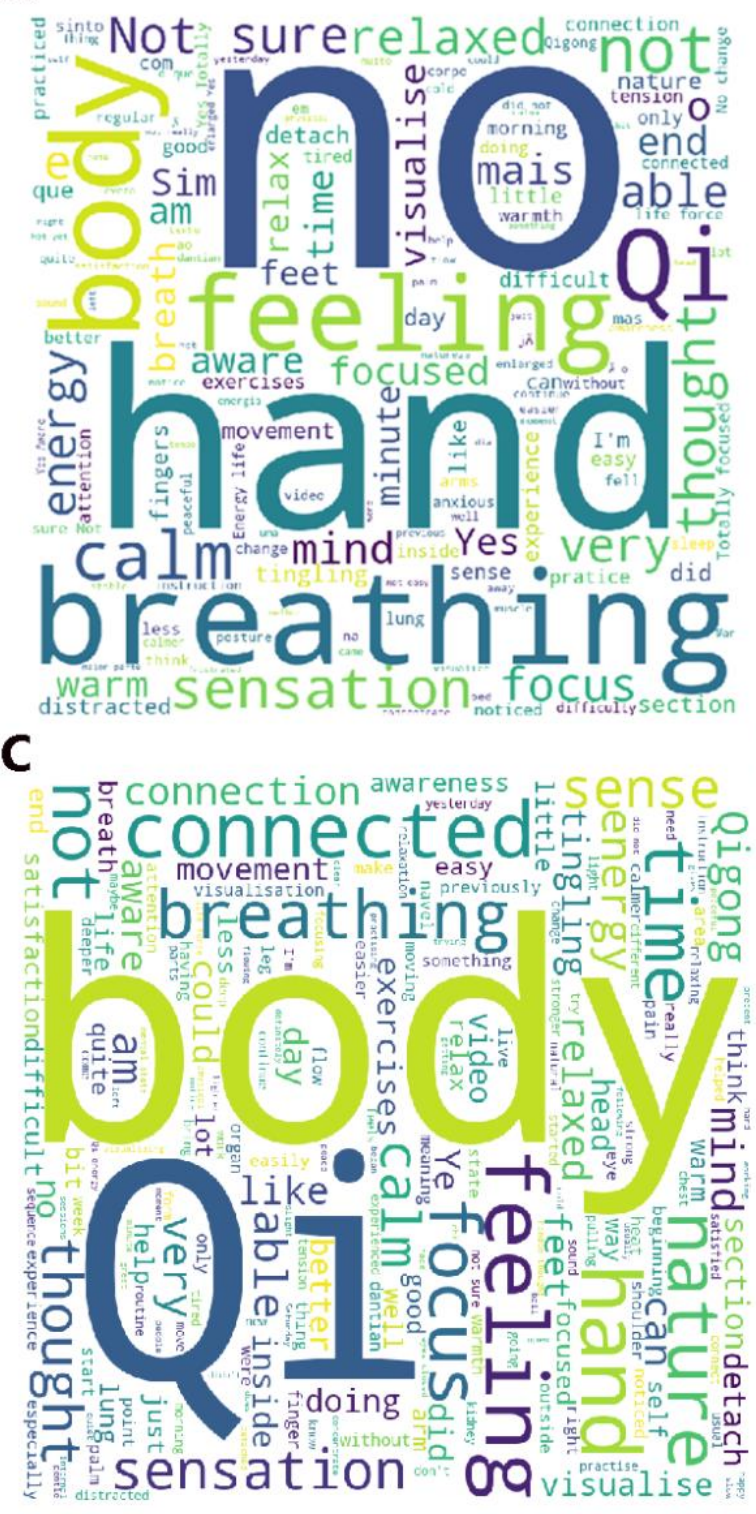

B

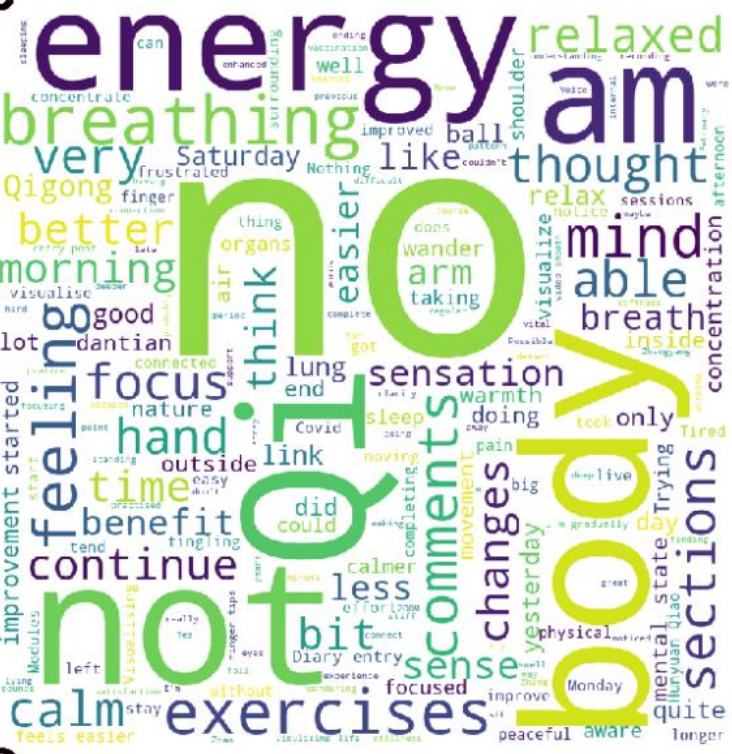

D

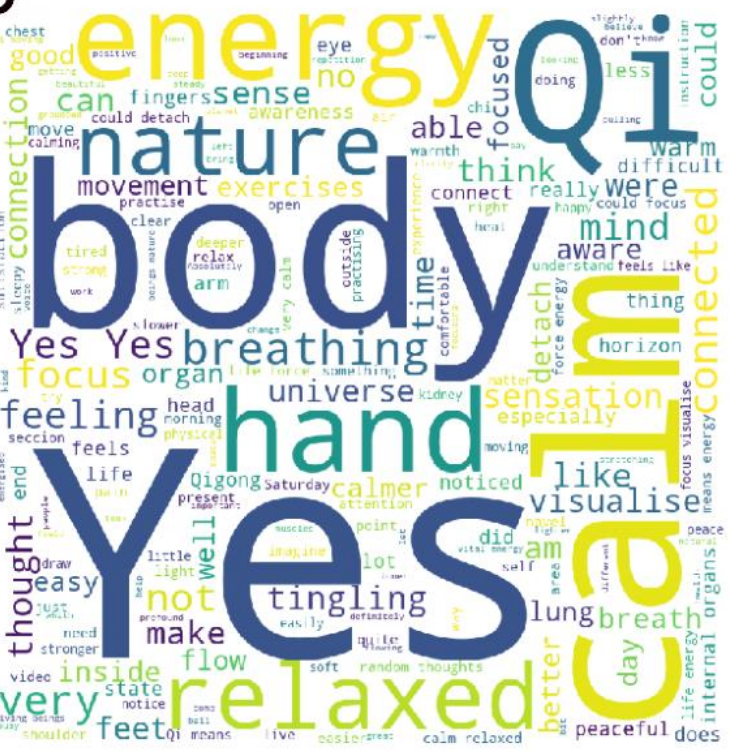

Figure 5 Wordcloud produced from the top 100 frequent words of cluster (A)1, (B)2, (C)3, (D)4 

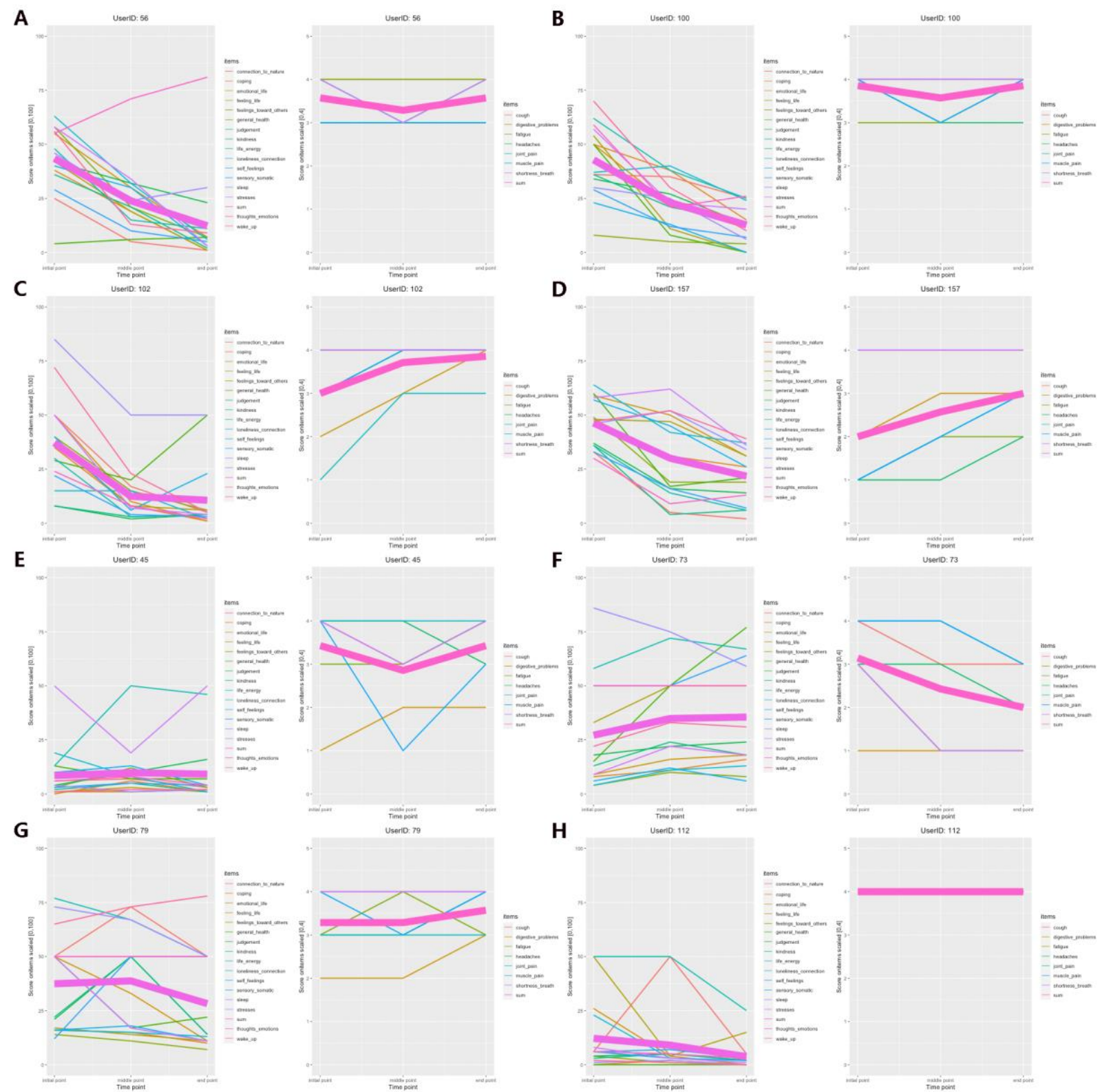

Figure 6 - Score changes of the baseline questionnaire items over time for the participant IDs (A) 56, (B) 100, (C) 102, (D) 157, (E) 45, (F) 73, (G) 79, (H) 112 